\title{
Two-dimensional nucleation and growth mechanism explaining graphene oxide structures
}

\author{
Manh-Thuong Nguyen* \\ The Abdus Salam International Centre for Theoretical Physics, Strada Costiera 11, I-34151 Trieste, Italy \\ Rolf Erni \\ Electron Microscopy Center, Empa, Swiss Federal Laboratories for Materials Science and Technology, CH-8600 Dübendorf, Switzerland \\ Daniele Passerone \\ nanotech@surfaces, Empa, Swiss Federal Laboratories for Materials Science and Technology, CH-8600 Dübendorf, Switzerland
}

(Received 26 April 2012; revised manuscript received 7 August 2012; published 5 September 2012)

\begin{abstract}
Using first-principles calculations accompanied by experimental data, we present a study on the structural evolution of graphene during oxidation to form partially oxidized graphene oxide. Our simulations show that local distortions in graphene induced by bond formation with adatoms indirectly lead to a "symbiotic" behavior of co-adsorbed oxygen atoms. Namely, the adatoms tend to mutually stabilize the adsorption and possibly migrate on graphene in groups of some small number of atoms. These theoretical results provide an explanation for the experimentally observed structures of graphene oxide.
\end{abstract}

DOI: 10.1103/PhysRevB.86.115406

PACS number(s): 81.15.Aa, 31.15.A-, 68.65.Pq

\section{INTRODUCTION}

Graphene is more and more considered an excellent candidate for future electronic nanodevices. ${ }^{1-3}$ Besides the intrinsic, highly promising physical properties of this intact two-dimensional allotrope of carbon, new properties induced by decorating graphene with adatoms and molecules have captured attention as a controlled decoration of graphene might outline a way of tailoring a band gap in graphene devices. ${ }^{1,4,5}$ Adatoms, oxygen in particular, can change the electronic structure and conductivity of graphene making graphene a semiconductor or an insulator, depending on the degree of oxidation. $^{6-9}$ Yet, in order to understand and predict the properties of modified graphene a theoretical approach is mandatory. In particular, for the case of graphene oxide (GO), where graphene is functionalized with oxygen atoms, theoretical models are often based on "artificial" oxygen arrangements, even with ordered structures. Ordered oxygen atoms in theoretical models of GO can result from considering a too small unit cell combined with periodic boundary conditions, ${ }^{10-12}$ which might impose an unrealistic symmetry in the model. Such long-range ordered arrangements of oxygen atoms on graphene miss, however, experimental evidence. In agreement with x-ray absorption data, ${ }^{13}$ transmission electron microscopy (TEM) has revealed that GO consists of disordered areas of high oxygen functionalization that surround patches of pristine graphene, ${ }^{14,15}$ as shown in Fig. 1. These experimental observations thus suggest that oxygen atoms tend to aggregate in denser groups rather than being separate. Moreover, direct observation of the dynamics of adatoms on a sheet of graphene has recently been possible due to advancements in atomicresolution aberration-corrected TEM. ${ }^{14,16}$ TEM observations supported by theoretical calculations revealed that covalently bound adsorbates can diffuse on graphene in a time scale of seconds. ${ }^{16}$ Hence, as these adsorbates can be relatively mobile at room temperature and might even be prone to desorption, the issue of ordered GO models can easily be a subject of dispute. Specifically, the thermal instability of the adsorbates may hinder decorated graphene from having predictable characteristics. A very rational question is: How can we understand the experimental observation of GO using a theoretical approach? This question motivates us to take a closer look at the formation of GO and the fundamental mechanism that governs the initial arrangement of oxygen atoms on graphene. In this work we employed density functional theory (DFT) calculations in order to rationalize the formation of local structures of GO. We show that energetics drives oxygen adatoms to occupy neighboring graphene sites, and that the oxidation of graphene thus occurs in patches following a two-dimensional "nucleation-and-growth" mechanism. The rest of this paper is organized as follows: In Sec. II we address the theoretical approaches and the graphene unit cell used and in Sec. III we present results and a discussion of the formation of oxygen islands of increasing sizes on graphene. The findings are finally summarized in Sec. IV.

\section{METHODS}

DFT simulations were carried out with the CP2K package, ${ }^{17}$ using the PBE exchange-correlation functional ${ }^{18}$ corrected with a semiempirical van der Waals energy. ${ }^{19}$ The Gaussian and plane-wave (GPW) hybrid basis set is employed: The localized Gaussian-based TZV2P for $\mathrm{C}$ and $\mathrm{O}$, and plane waves with a cutoff of $280 \mathrm{Ry}$ for the charge density. The ions and core electrons are described through norm conserving GTH pseudopotentials. ${ }^{20}$ To evaluate the activation energy in the migration processes of adsorbates on graphene, we used the socalled climbing image nudged elastic band (NEB) method. ${ }^{21}$ Replicas in a band are initialized by linearly interpolating the coordinates of the initial state and the final state. In this work we used a fairly large $51.2 \times 44.4 \times 30 \AA^{3}$ orthorhombic unit cell containing 864 carbon atoms and oxygen adsorbates.

\section{RESULTS AND DISCUSSION}

The general properties of a simulated agglomerate of oxygen adatoms, of the kind studied in this paper, are shown in Fig. 2. On the basis of the existing literature ${ }^{10,22}$ and of 


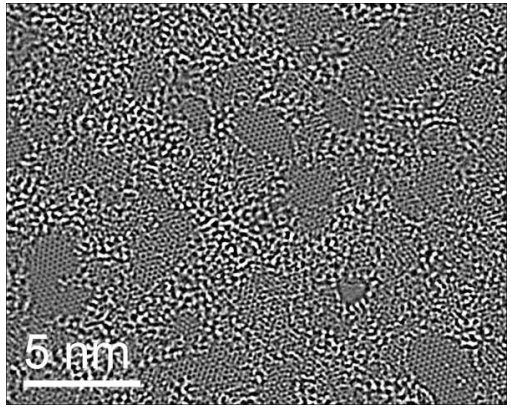

FIG. 1. TEM phase image revealing patches of pristine graphene that surround disordered areas of high oxygen functionalization. Phase of an exit-plane wave reconstructed from a focal series of 12 members. The focal range of the series covers +11.9 to $+3.9 \mathrm{~nm}$ with a third- and fifth-order spherical aberration of $-14 \mu \mathrm{m}$ and $+8 \mathrm{~mm}$ using a monochromated illumination at $80 \mathrm{keV}$ electron energy that provides a focal spread of about $1.2 \mathrm{~nm}$.

energetic considerations, we focus in this paper on epoxy groups, namely three-membered rings involving an oxygen atom and two carbon atoms. The figure shows an example of an agglomerate of 48 epoxy groups adsorbed on either side of the graphene sheet. As pointed out by several authors (see, e.g., Li et al. ${ }^{22}$ ), an epoxy group stretches the underlying C-C bond and "pulls" the carbon bonding partners, thus causing a corrugation in the graphene sheet. Moreover, additional corrugation is observed around the island (of the order of 0.1-0.3 $\AA$ ). Such modifications and relaxation of the graphene sheet could be responsible for the creation of grain boundaries among the GO patches, hindering the coalescence of such patches into a single compact island (see Fig. 1). The lower panel of Fig. 2 shows a lateral view of the GO island, where the strong buckling of carbon is observed. The adsorption on both sites has the advantage of reducing the stress of the graphene sheet upon buckling.

We now consider the elementary processes leading to the formation of the afore described bigger island. As a starting point we consider the adsorption and diffusion of one oxygen atom on graphene and refer to this system as a "monomer" case. Systems of single oxygen atoms on graphene are well understood. ${ }^{16,23,24}$ From an electronic structure point of view, a bridge site, that is, on top of a carbon-carbon bond, provides
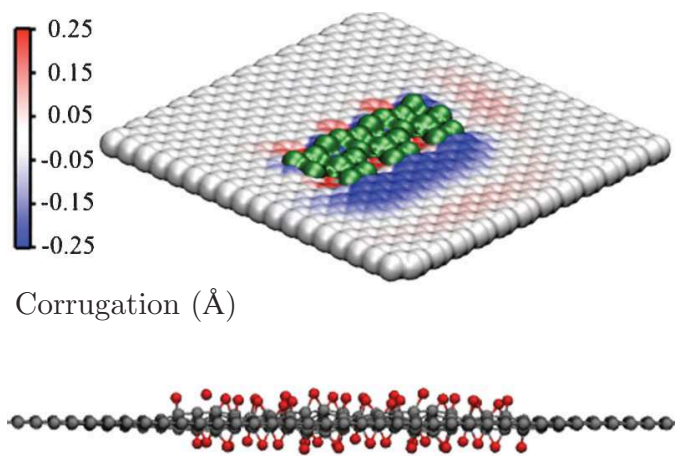

FIG. 2. (Color online) Simulation model with 864 carbon atoms and an island of 48 oxygen atoms (in green). The corrugation profile beneath and around the adatoms is shown, together with the lateral view showing the disordered agglomerate of epoxy groups. the stable position for a single (divalent) oxygen atom on graphene, resulting in the formation of an epoxy group. The binding energy, which is defined in this work as the difference in energy between the bound and the separated systems (oxygen is considered as atomic in the triplet state), amounts to $-1.8 \mathrm{eV}$, showing that the adsorbate cannot be desorbed at room temperature. A NEB calculation predicts that the activation energy needed to initiate the dynamics of oxygen to take place is just about $0.6(3) \mathrm{eV}$, consistent with a previous calculation. ${ }^{25}$ This reveals that the oxygen atom cannot be desorbed but that the adatom can easily migrate on graphene at room temperature. If the hopping rate of oxygen is given (within harmonic transition state theory) by Vineyard's equation $k^{\mathrm{HTST}}=v_{0} e^{-E_{a} / k T}{ }^{26}$ where $E_{a}$ is the static barrier height, and the prefactor $\nu_{0}$ which can be computed through vibrational analysis typically varies between $10^{12}$ and $10^{14} \mathrm{~s}^{-1}$, the adatom will undergo rather fast transitions on a time scale that can range from seconds to milliseconds at room temperature, but increase by several orders of magnitude upon increase of the barrier by, say, $0.1 \mathrm{eV}$. Moreover, the rate from transition state theory is an upper limit to the real rate. This estimate is in agreement with earlier TEM experiments where however the electron irradiation in the electron microscope can accelerate the dynamics. ${ }^{16}$ It is also instructive to know that the carbon bonding partners of the oxygen atom on the bridge position are shifted approximately $0.2 \AA$ out off-plane compared to their initial positions. This can be reasoned by considering the hybridization of the carbon atoms whose atomic orbitals are no longer the flat $s p^{2}$ of graphene, while an amount of about $0.7 e$ is transferred from graphene to the adatom as predicted by the Bader analysis. ${ }^{27}$ The elastic effect of the membrane on the adhesion of the system is further examined by repeating the geometry optimization without allowing graphene to relax. It turns out that the system becomes $0.9 \mathrm{eV}$ less stable despite the $0.7 \mathrm{eV}$ energy cost to deform graphene from the nonoxygen-adsorption (flat) to the oxygen-adsorption (crinkled) shape.

As interadsorbate interactions are a necessary angle of the present work, we introduced an additional oxygen atom on the other side of graphene and looked at the total energy when the two adatoms are moving towards each other. Geometry optimizations reveal that local minima are always obtained when both atoms are at the bridge sites except for two adjacent ones. Several configurations ${ }^{28}$ are examined sampling the structural stability when the two adatoms are separated by 2, 3, 4, . graphene carbon atoms [see Figs. 3(a)-3(d)]. Our calculations provide evidence that the most stable structure is realized when the two adatoms are separated by one single bridge site and both are on the same hexagonal ring on opposite sides of graphene. ${ }^{28}$ The outstanding stability of this configuration [Fig. 3(b)] can, in part, be attributed to the local distortion and by considering the lack of aromaticity of decorated graphene. To understand the first factor we again relax the structures of the most and the second most stable configurations, namely, $(2,2)$ and $(2,1)$, with fixed graphene coordinates. Doing so, the relative energy between them substantially decreases from $0.09 \mathrm{eV}$ (fully relaxed) to $0.05 \mathrm{eV}$ (graphene constrained). Additionally, as pointed out elsewhere, ${ }^{10}$ the lattice constant of graphene increases with oxygen coverage due to the depletion of the bonding 


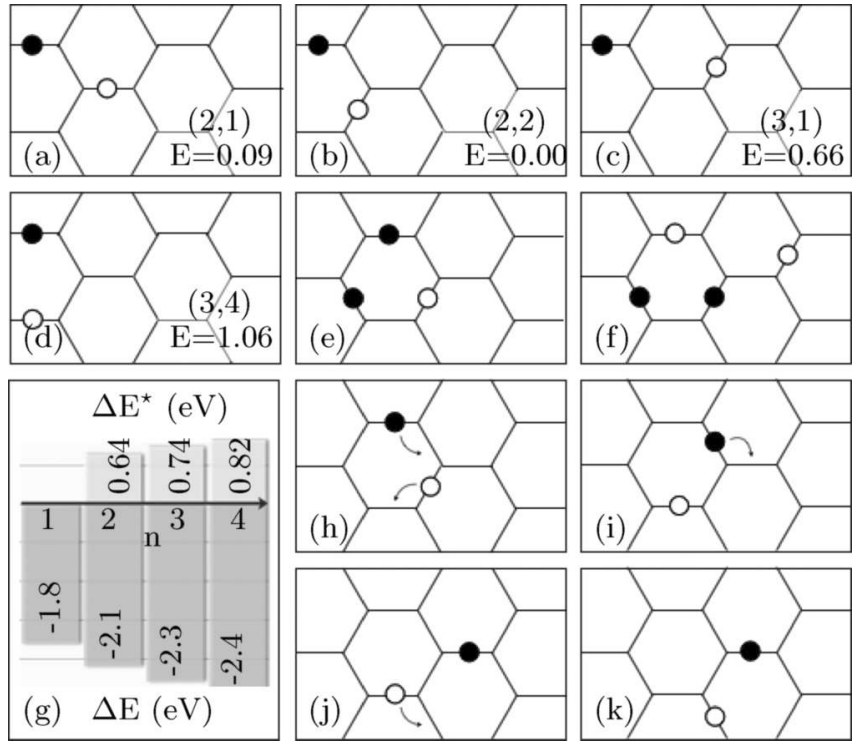

FIG. 3. (a)-(d) Some configurations of two oxygen atoms on graphene: • (०) denotes an atom above (underneath) graphene; $(2,1)$, $(2,2), \ldots$ imply relative positions between the two atoms (see the text), $(2,1)$ and $(2,2)$ are the most stable structures. (e) and (f) Lowest energy configurations of investigated ones when more adatoms are added. (g) Energy difference when $(n-1)$ adatoms are in the center of the cell, 1 adatom is in a corner of the cell box, and when $n$ adatoms are in the cell center $\Delta E^{\star}$; adsorption energy per oxygen atom $\Delta E$ against number of oxygen atoms $n$. (h)-(k) A possible diffusion mechanism of two adatoms: They simultaneously move in a hexagon (h) and then one-by-one move to a neighboring hexagon (i)-(k).

$p_{z}(\pi)$ orbitals of carbon. Geometric modification therefore plays a certain role. As for the aromaticity, from all the investigated configurations, the $(3,4)$ configuration [Fig. 3(d)] accommodates two unpaired $p_{z}$ electrons, which essentially explains its low stability. Hence, both distortions and the configuration of the electrons are important factors controlling the stability and structure of adatoms on graphene.

Comparing the case of the dimer with the monomeric case, the binding energy per oxygen atom for the most stable structure is $-2.1 \mathrm{eV}$, which is significantly higher than the one in case of the monomer. This improvement of conformational stability suggests that graphene-mediated interadsorbate interaction leads to a "symbiosis" between adatoms. Moreover, given that single oxygen adatoms can easily diffuse on graphene, they can reach the favorable dimeric structure where they are trapped in the dimeric configuration. The reason why we have considered the dimer with oxygen atoms on different sides of graphene is that in our TEM experiment (Fig. 1) oxygen adatoms are deposited on both sides. Furthermore, the most stable oxygen dimer appears to be one of such structures. To be specific, we reexamine the adhesion of atomic oxygen on graphene by considering some illustrative structures, namely, $(2,1),(2,2),(3,4)$ where both adatoms are on the same side. It appears that the systems become less stable by as much as by $0.16-0.20 \mathrm{eV}$, except for the $(3,4)$ case [however, the same-side $(3,4)$ is sill much less stable than the same-side $(2,2)]$, where the "same-side" structure is more stable with respect to the corresponding "opposite-side" configuration. This same-side $(3,4)$ structure is indicated by Ref. 22 as a

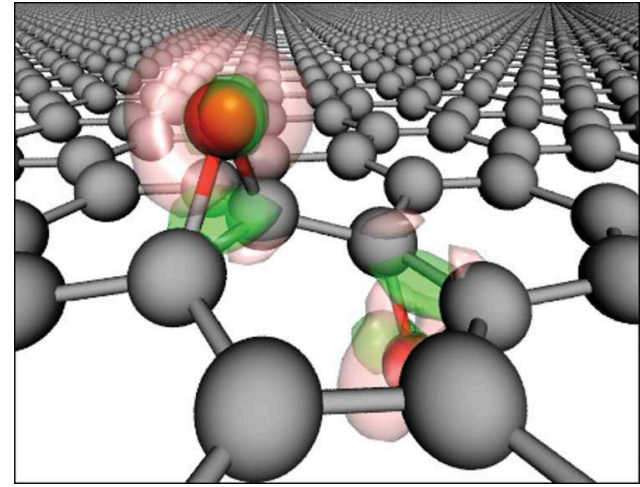

FIG. 4. (Color online) Electron density difference in the dimer $(2,2)$ : Isosurface at 0.015 a.u., accumulation in pink and depletion in green.

possible precursor for nanographene zippering. If graphene is available to oxygen from both sides like in this case, the most stable opposite-side $(2,2)$ structure makes the nucleation of zippering chains less probable. Thus, even in case that long zippering epoxy chains may be particularly stable, the formation of such chains may be hindered by the presence of concurrent nucleation processes. Long-range electrostatics due to graphene-oxygen charge transfer (see Fig. 4) is among possible dominant factors accounting for the energy difference between same-side and opposite-side oxygen dimers.

A central question concerns the thermal stability of the adatoms and whether thermal energy can separate them from a configuration that is stabilized by the interadsorbate interaction. To address this issue we performed NEB calculations to find a path such that an adatom can practically escape from the other. Suppose that while one oxygen atom is sitting in its position the other hops from a bridge site to an adjacent (bridge) site, it subsequently moves backwards to its initial position or continues its diffusion path. Several possibilities should, of course, be taken into account. ${ }^{28}$ It is intriguing to note that the transition between the two lowest minima $[(2,1),(2,2)]$ can be very fast since the corresponding energy barrier is just $0.3 \mathrm{eV}$. The most energy demanding step breaking the neighborhood between the two atoms is to transfer the moving atom from its initial position to a carbon position that separates the oxygen atoms by one more unit length [e.g., from $(2,1)$ to $(3,1)]$. For such a transition, the diffusion energy barrier is about 1.0 $\mathrm{eV}$, which is higher than the one of isolated oxygen adatoms. Additional molecular dynamics simulations ${ }^{28}$ are in excellent agreement with our NEB calculations. This finding emphasizes that the presence of an additional adsorbate reduces the mobility of its co-adsorbed partner, reminiscing the stability and dynamics of trapped functional groups on graphene. ${ }^{16}$ It can be intuitively interpreted by looking back at the "potential energy surface" (PES) of the dimer at graphene in which a notably low minimum is observed. This surface is inherently different from the single adatom PES of equal minima.

Last but not least, it is also possible that the co-adsorbed partner of the diffusing atom can follow it, leading to the migration of the dimer. Adatoms can move in one-by-one step fashion. In the previous picture this would correspond to the situation where right after the second adatom jumps 
the first adatom moves onward. Alternatively, simultaneous two-adatom diffusion can take place, too. The two adatoms can move from $(2,1)$ or $(2,2)$ to equivalent sites in the neighboring hexagons. Otherwise, a mixed one-by-one and simultaneously triggered diffusion step is also possible. In Figs. 3(h)-3(k) we show such a diffusion pathway underlying the migration of the two adatoms between any two carbon hexagons: Two adatoms simultaneously move to adjacent bridge sites on the same hexagon, then one-by-one move to a neighboring carbon ring. Our NEB calculations unveil that this group of adatoms can migrate with a barrier of $1.0 \mathrm{eV}$. Hence, considering the diffusion barrier of an isolated oxygen atom, the oxygen pair is clearly less mobile. This result brings forward the physical picture of the adsorption mechanism of two oxygen adatoms on graphene which isolated can diffuse until to the moment that they trap each other and stabilize the adsorption of each other. Of course, a correlated diffusion mechanism of the two oxygen atoms cannot be ruled out, nonetheless this would occur at a much lower rate.

Consider that there exists a (stable) dimeric conformation in some region of graphene, what would happen if we add a third oxygen atom to the system? As the number of possible conformations is very large, we simplify our models based on the following assumption. Two initial oxygen atoms are in one of the two conformations $(2,1)$ or $(2,2)$, a more stable structure is obtained if the third atom is added in such a way that at least one of the two oxygen atoms forms a $(2,1)$ or $(2,2)$ structure with the new adatom regardless of whether they are on the same side or opposite sides of graphene. Investing 20 trimeric configurations ${ }^{28}$ brings us to the conclusion that the adhesion of the trimer oxygen cluster on graphene is enhanced compared to the dimer case. The adsorption energy per oxygen now is $-2.3 \mathrm{eV}$ corresponding to the case of three adatoms decorating the same carbon hexagon. The enhancement of the stability of oxygen with respect to the adatom cluster size in our calculations is in reasonable agreement with the increasing tendency of the binding energy of oxygen with respect to the adatom coverage. ${ }^{10} \mathrm{We}$ also notice that the breakdown of the $\mathrm{C}-\mathrm{C}$ bonds in the epoxy groups of this trimer [Fig. 3(e)] is unlikely since it requires a considerable distortion of graphene on both sides, thus hindering a possible "unzipping and cutting" mechanism of three oxygen atoms which is found in a system of three oxygen atoms at the same side of graphene. ${ }^{29}$ Next we consider a few tetrameric cases. Starting from the most stable trimeric structure previously found [see Fig. 3(e)], we add one more oxygen atom. Despite that only six configurations are investigated, ${ }^{28}$ the adhesion energy per adatom is higher than the trimeric case [see Fig. 3(f)].

Finally, we examined considerably larger islands based on the most stable building blocks found above. We built 16membered $^{28}$ and 48-membered (shown in Fig. 2) islands of oxygen on graphene and fully optimized the structures, and found a binding energy per oxygen atom of -2.6 and $-2.7 \mathrm{eV}$, respectively.

One might question the reliability of our calculated binding energies due to the Gaussian basis set superposition errors ${ }^{30}$ and possible density functional dependence, nevertheless, additional plane-wave DFT calculations within local density approximations show that this trend is confirmed. ${ }^{28}$
Our results show a tendency of oxygen adatoms to aggregate in dense groups, that is, for a given number of adatoms the total energy is significantly lowered when adatom clusters are formed, thus consistent with the adhesion energies per oxygen (see Fig. 3). In agreement with previously published micrographs, ${ }^{14,15}$ the TEM data of GO shown in Fig. 1 reveal patches of pristine graphene that are surrounded by disordered areas of high oxygen functionalization. An important aspect about these experimental results is that the oxygen functionalization is confined to well defined areas. On the basis of the DFT results of the monomer, dimer, trimer, and tetramer oxygen configurations on graphene, we can explain the structure of GO as emerging from (stable) clusters of oxygen atoms on graphene which attract further functionalization. The fact that the adsorption energy per atom increases with increasing size of the oxygen cluster on graphene outlines a trend: Oxygen atoms tend to occupy adjacent sites on graphene resulting in clusters of high oxygen functionalization. Furthermore, oxygen clusters seem to become less mobile when their size is larger. A graphene area that hosts an oxygen cluster is energetically attractive for isolated mobile oxygen atoms. This supports the idea of a two-dimensional nucleation-and-growth mechanism which underlies the formation of GO. The critical radius of a nucleus essentially corresponds to a single oxygen atom on graphene. Such isolated nuclei mutually attract each other, stabilize themselves, and keep on growing on further oxidation. The functionalized areas will further expand until they form a network of high functionalization that surrounds remaining patches of pristine graphene. The driving force for this mechanism is found in minimizing the distortion energy on adding oxygen atoms to graphene as well as in establishing an electronic structure that minimizes the occurrence of unpaired $p_{z}$ electrons in partially decorated graphene. Moreover, having remaining patches of pristine graphene that are surrounded by highly functionalized areas implies that the properties of GO emerge from a nanocomposite material. This is in contradiction with Lu et al. ${ }^{12}$ who conclude that even partially oxidized graphene forms a homogeneous material. Based on our results, however, we find that controlling the properties of GO by adjusting the degree of oxidation means to balance the areas of pristine graphene against highly functionalized areas, which on a nanometer scale exhibit different properties.

Our results, which are partially contradicting to the results by $\mathrm{Lu}$ et al., ${ }^{12}$ have to be put in perspective with calculations performed by Wang et al., ${ }^{11}$ who state that for oxygen coverage less than 0.5 the homogeneous phase may be favored upon the inhomogeneous one. However, although a large unit cell would be needed to support their statement, Wang et al. ${ }^{11}$ base their homogeneous model for low coverage on periodic repetitions of dense islands. A too small cell periodically repeated, however, does not allow a thorough evaluation about the presence of different phases and inhomogeneities. By aid of our large unit cell, we optimized the graphene sheet with 108 most stable $(2,2)$ dimers (accordingly, a 0.25 coverage) arranged in a periodic (ordered) fashion and in a disordered arrangement in which these oxygen atoms are grouped in patches as displayed in Fig. 5. The latter appears to be $101.9 \mathrm{eV}$ more stable than the former.

Periodic structures based on larger subunits can improve the stability of such arrangements. We tested this concept by 


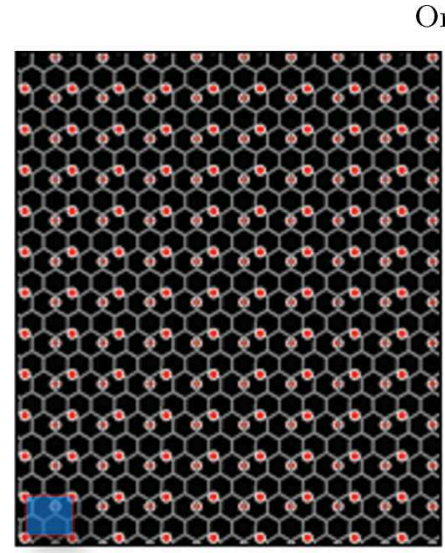

(a) $\mathrm{E}=+101.9 \mathrm{eV}$
Order

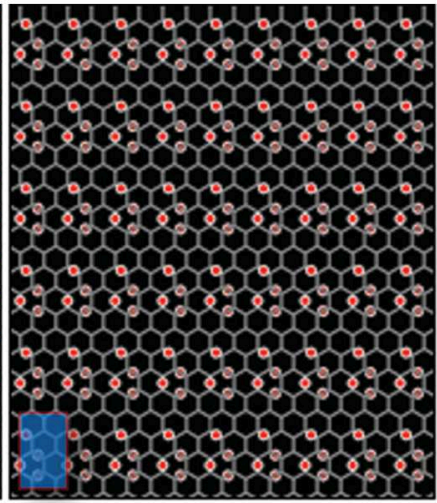

(b) $\mathrm{E}=+67.1 \mathrm{eV}$
Disorder

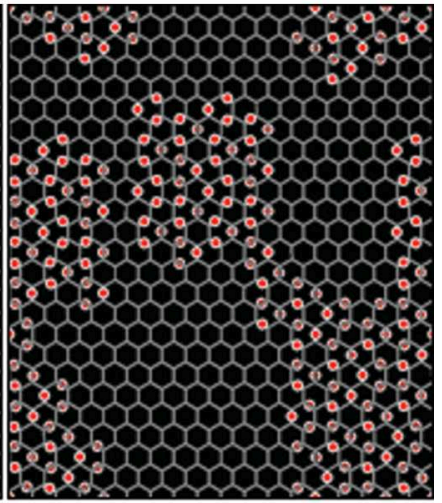

(c) $\mathrm{E}=0.0 \mathrm{eV}$

FIG. 5. (Color online) Order or disorder? 216 oxygen atoms on a 864 carbon atom periodic graphene sheet in an ordered arrangement by (a) 108 most stable $(2,2)$ dimers (the blue rectangle indicates one of 108 "subunits"), (b) 54 tetramers, and (c) a disordered structure, the latter shows superior stability.

modeling a supercell based on a periodic lattice of tetrameric elements [see Fig. 5(b)]. This structure is $67.1 \mathrm{eV}$ less stable than the disordered one. The energy gain associated with the increase of the subunit size from dimeric to tetrameric is essentially based on short range effects. Interestingly, such energy gain is not larger (it is actually smaller!) than the one expected by adding up the energy gain difference between isolated dimers and tetramers as deduced from Fig. 3(g). Since we did not include basis set superposition error correction in our estimates, we can only interpret this fact qualitatively, and note that no long-range order energy gain is observed in the simulated superstructures upon increase of the subunit size. We did not investigate larger subunits, but we can speculate that, as soon as an island gets larger and larger, it is kinetically unlikely that periodic repetitions of such an island are formed. Rather, for a given coverage, as confirmed by experiment, and compatibly with our calculations, a large, disordered aggregated cluster surrounded by pristine graphene regions appears to be a stable arrangement for adsorbed atomic oxygen.

\section{CONCLUSION}

In conclusion, by using DFT calculations to derive stable configurations of monomer, dimers, trimers, and tetramers of oxygen atoms on graphene we show that with increasing number of oxygen atoms configurations are energetically favorable when the oxygen atoms form small clusters on graphene occupying adjacent sites on both sides of a graphene flake. This trend provides the basis to claim that oxidation of graphene occurs in a 2D nucleation-and-growth mechanism. Through buckling and corrugation, the creation of islands leads to structural (and electronic) modifications of the pristine graphene layer. The energy per adsorbed oxygen atom increases with the dimension of the island: Agglomeration of oxygen atoms in dense island thus appears to be more stable than a homogeneous arrangements of the oxygen atoms. The underlying mechanism can explain the observed inhomogeneous structure of $\mathrm{GO}$ which consists of pristine graphene patches surrounded by areas of high oxygen functionalization. It is expected that the agglomeration of adatoms on graphene is also a valid picture for other species decorating graphene.

\section{ACKNOWLEDGMENTS}

M.-T.N. gratefully acknowledges computer support by CINECA (project N. HP10BRNG9S). R.E. acknowledges K. Erickson and A. Zettl (UC Berkeley) for providing the GO sample. D.P. acknowledges financial support by the Swiss National Science Foundation (SNSF) and computer support by the Swiss National Supercomputing Centre (CSCS). *mtnguyen@ictp.it

${ }^{1}$ A. K. Geim and K. S. Novoselov, Nat. Mater. 6, 183 (2007).

${ }^{2}$ A. H. CastroNeto, F. Guinea, N. M. R. Peres, K. S. Novoselov, and A. K. Geim, Rev. Mod. Phys. 81, 109 (2009).

${ }^{3}$ S. Malik, A. Vijayaraghavan, R. Erni, K. Ariga, I. Khalakhan, and J. P. Hill, Nanoscale 2, 2139 (2010).

${ }^{4}$ K. Novoselov, A. Geim, S. Morozov, D. Jiang, M. Katsnelson, I. Grigorieva, S. Dubonos, and A. Firsov, Nature (Londoon) 438, 197 (2005).

${ }^{5}$ J. J. Hernandez Rosas, R. E. Ramirez Gutierrez, A. EscobedoMorales, and E. Chigo Anota, J. Mol. Model. 17, 1133 (2011).
${ }^{6}$ P. O. Lehtinen, A. S. Foster, A. Ayuela, A. Krasheninnikov, K. Nordlund, and R. M. Nieminen, Phys. Rev. Lett. 91, 017202 (2003).

${ }^{7}$ K. T. Chan, J. B. Neaton, and M. L. Cohen, Phys. Rev. B 77, 235430 (2008).

${ }^{8}$ F. Schedin, A. K. Geim, S. V. Morozov, E. W. Hill, P. Blake, M. I. Katsnelson, and K. S. Novoselov, Nat. Mater. 6, 652 (2007).

${ }^{9}$ A. Bagri, C. Mattevi, M. Acik, Y. J. Chabal, M. Chhowalla, and V. B. Shenoy, Nat. Chem. 2, 581 (2010).

${ }^{10}$ J. Ito, J. Nakamura, and A. Natori, J. Appl. Phys. 103, 113712 (2008). 
${ }^{11}$ L. Wang, Y. Y. Sun, K. Lee, D. West, Z. F. Chen, J. J. Zhao, and S. B. Zhang, Phys. Rev. B 82, 161406(R) (2010).

${ }^{12}$ N. Lu, D. Yin, Z. Li, and J. Yang, J. Phys. Chem. C 115, 11991 (2011).

${ }^{13}$ S. Saxena, T. A. Tyson, and E. Negusset, J. Phys. Chem. Lett. 1, 3433 (2010).

${ }^{14}$ K. Erickson, R. Erni, Z. Lee, N. Alem, W. Gannett, and A. Zettl, Adv. Mater. 22, 4467 (2010).

${ }^{15}$ D. Pacile, J. C. Meyer, A. F. Rodríguez, M. Papagno, C. GómezNavarro, R. S. Sundaram, M. Burghard, K. Kern, C. Carbone, and U. Kaiser, Carbon 49, 966 (2011).

${ }^{16}$ R. Erni, M. D. Rossell, M.-T. Nguyen, S. Blankenburg, D. Passerone, P. Hartel, N. Alem, K. Erickson, W. Gannett, and A. Zettl, Phys. Rev. B 82, 165443 (2010).

${ }^{17}$ J. Van de Vondele, M. Krack, F. Mohamed, M. Parrinello, T. Chassaing, and J. Hutter, Comput. Phys. Commun. 167, 103 (2005).

${ }^{18}$ J. P. Perdew, K. Burke, and M. Ernzerhof, Phys. Rev. Lett. 77, 3865 (1996).

${ }^{19}$ S. Grimme, J. Antony, S. Ehrlich, and H. Krieg, J. Chem. Phys. 132, 154104 (2010).

${ }^{20}$ S. Goedecker, M. Teter, and J. Hutter, Phys. Rev. B 54, 1703 (1996).
${ }^{21}$ G. Henkelman, B. Uberuaga, and H. Jonsson, J. Chem. Phys. 113, 9901 (2000).

${ }^{22}$ J. L. Li, K. N. Kudin, M. J. McAllister, R. K. Prudhomme, I. A. Aksay, and R. Car, Phys. Rev. Lett. 96, 176101 (2006).

${ }^{23}$ R. J. W. E. Lahaye, H. K. Jeong, C. Y. Park, and Y. H. Lee, Phys. Rev. B 79, 125435 (2009).

${ }^{24}$ T. O. Wehling, M. I. Katsnelson, and A. I. Lichtenstein, Phys. Rev. B 80, 085428 (2009).

${ }^{25}$ A. M. Suarez, L. R. Radovic, E. Bar-Ziv, and J. O. Sofo, Phys. Rev. Lett. 106, 146802 (2011).

${ }^{26}$ A. F. Voter, F. Montalenti, and T. C. Germann, Annu. Rev. Mater. Res. 32, 321 (2002).

${ }^{27}$ R. F. W. Bader, Atoms in Molecules-A Quantum Theory (Oxford University Press, Oxford, 1990); W. Tang, E. Sanville, and G. Henkelman, J. Phys.: Condens. Matter 21, 084204 (2009) [http://theory.cm.utexas.edu/bader/].

${ }^{28}$ See Supplemental Material at http://link.aps.org/supplemental/ 10.1103/PhysRevB.86.115406 for all the investigated oxygen dimers, trimers, tetramers, molecular dynamics simulations, and plane-wave DFT calculations.

${ }^{29}$ T. Sun and S. Fabris, Nano Lett. 12, 17 (2012).

${ }^{30}$ S. Boys and F. Bernardi, Mol. Phys. 19, 553 (1970). 